\title{
Two-Phase Multi-Objective Evolutionary Approaches for Optimal Generation Scheduling with Environmental Considerations
}

\author{
Dapeng Li, Sanjoy Das, and Anil Pahwa \\ Department of Electrical and Computer Engineering \\ Kansas State University \\ Manhattan, KS 66502 \\ Email: \{dapeng, sdas, pahwa\}@ksu.edu
}

\begin{abstract}
This paper presents novel two-phase multi-objective evolutionary approaches for solving the optimal generation scheduling problem with environmental considerations. Two different multi-objective evolutionary algorithms (MOEA) based on Strength Pareto Evolutionary Algorithm 2 (SPEA2) and Archived Multi-objective Simulated Annealing (AMOSA) are presented in the paper. In the first phase, this approach formulates the hourly optimal generation scheduling problem as a multi-objective optimization problem which simultaneously minimizes operation cost and emission, while satisfying constraints such as power balance, spinning reserve and power generation limits. Results of the first phase are compared and SPEA2, which provided better results, is used for the second phase to obtain the optimal schedules for the 24 hours. The minimum up/down time and ramp up/down rate constraints are incorporated in the second phase. A case study for a 10-unit test system is carried out to illustrate the application and the effectiveness of the proposed approach.
\end{abstract}

\section{INTRODUCTION}

The principle objective of optimal generation scheduling problem for power utilities is to schedule the generation units effectively over a given time horizon while meeting forecasted load demand and a variety of operating constraints, such as spinning reserve requirements, minimum up/down time constraints, etc. When the only goal of scheduling the generation units is to minimize operation cost, this problem is usually referred to as the well-known unit commitment problem. Due to increased concern for environmental protection, U.S. electric power plant operators have the obligation to reduce atmospheric emissions of the thermal power plants, such as sulfur dioxide $\left(\mathrm{SO}_{2}\right)$ and nitrogen oxides $\left(\mathrm{NO}_{\mathrm{x}}\right)$, beyond the levels called for in the Clean Air Act Amendments of 1990 (CAAA90) [1]. As a short-term alternative to achieve the emission targets without investment for new pollutant cleaning equipment, emission dispatching is an effective strategy to keep the emission below a certain level.
Generation scheduling taking both operation cost as well as emission into account, which is a multi-objective optimization problem, has been receiving increased attention due to environmental concerns. In the past, the widely-used method for multi-objective optimization is the weighted-sum method, which has several drawbacks [2]. A better option is to use multi-objective evolutionary algorithms (MOEA), which are more efficient and powerful alternative for multiobjective optimization to find many different Pareto-optimal solutions.

To obtain the Pareto-optimal solutions to the optimal generation scheduling problem for the whole given period ( $T$ hours), a two-phase multi-objective evolutionary approach based on application of Strength Pareto Evolutionary Algorithm 2 (SPEA2) [3] and Archived Multi-objective Simulated Annealing (AMOSA) [4] are investigated. In the first phase, both SPEA2 and AMOSA are used to get the hourly-optimal generation schedules for minimizing the operation cost and emission simultaneously, while satisfying the load balance, power limits and spinning reserve constraints. In the second phase, the minimum up/down time and ramp up/down constraints are included, and because of superior performance SPEA2 is selected to assemble the optimal schedules for the entire time interval of $T$ hours.

In the next section, SEPA2 and AMOSA are introduced briefly. Section III presents the mathematical formulation of the optimal generation scheduling problem. Section IV gives a detailed explanation of proposed approach and in section $\mathrm{V}$, numerical results are presented and discussed. Finally, conclusions are given in section VI.

\section{Muli-OBJECtive EVOLUTIONARY AlgORITHMS}

Multi-objective optimization always requires simultaneous handling of several conflicting objectives. Within the multiobjective optimization framework, it is not easy to distinguish a better solution from an inferior one [5]. This is because a solution that is better along one objective maybe worse along another objective. Hence, the notion of dominance can be 
applied directly in the multi-objective context to find tradeoff solutions between objectives. Given two solutions, $A$ and $B$, we say that $A$ dominates $B$ iff $(i) A$ is at least as good as $B$ along all the objectives, and (ii) $A$ is better than $B$ along at least one objective. Given a set of feasible solutions, the nondominated front consists of all those solutions that are not dominated by any other ones.

The Pareto front is the non-dominated front of the set of all feasible solutions. Evolutionary algorithms are one of the most common strategies for multi-objective optimization. There are two important goals in a multi-objective optimization [6]:

a. to find a set of solutions as close as possible to the Pareto-optimal front

b. to find a set of solutions as diverse as possible.

Among many MOEAs, NSGA-II and SPEA2 have received a lot of attention and become popular approaches. Moreover, A simulated annealing based multi-objective algorithms, AMOSA, is also a new and powerful optimization approach using archive to store all the nondominated solutions.

Many multi-objective optimization problems have equality or inequality constraints. In this paper, the concept of constrained-domination is used when solutions violate the constraints [6]:

A solution $A$ is said to constrained-dominate another solution $B$, if any of the following conditions is true.

1) $A$ is feasible and $B$ is not.

2) $A$ and $B$ are both infeasible, but $B$ has a smaller overall constraint violation.

3) $A$ and $B$ are both feasible and $A$ dominates $B$.

\section{A.Strength Pareto Evolutionary Algorithm 2}

SPEA2 is an improved version of the original SPEA. Compared with SPEA, the SPEA2 incorporates a fine-grained fitness assignment strategy, a density estimation technique, and an enhanced archive truncation method. Here, at the iteration $\mathrm{t}=0$, an initial population $\mathbf{P}_{0}$ of size $N$ and the empty archive $\mathbf{P}_{0}$ are created. After fitness values of individuals in $\mathbf{P}_{\mathrm{t}}$ and $\mathbf{P}_{\mathrm{t}}$ are evaluated, all nondominated individuals in $\mathbf{P}_{\mathrm{t}}$ and $\mathbf{P}_{\mathrm{t}}^{\prime}$ are moved to $\mathbf{P}_{\mathrm{t}+1}^{\prime}$. If size of $\mathbf{P}_{\mathrm{t}+1}^{\prime}$ exceeds $N$, then $\mathbf{P}_{\mathrm{t}+1}$ is reduced to size $N$ by means of the truncation operator. If the size of $\mathbf{P}_{\mathrm{t}+1}$ is less than $N$ then $\mathbf{P}_{\mathrm{t}+1}$ is filled with dominated individuals from $\mathbf{P}_{\mathrm{t}}$ and $\mathbf{P}_{\mathrm{t}}$. If the stopping criterion is satisfied, the set of decision vectors represented by the nondominated individuals in $\mathbf{P}_{t+1}$ are then set to $\mathbf{A}$ and the algorithm terminates. Otherwise, binary tournament selection is performed with replacement on $\mathbf{P}_{\mathrm{t}+1}$ in order to fill the mating pool and a new population $\mathbf{P}_{\mathrm{t}+1}$ is obtained by applying recombination and mutation operators to the mating pool. This procedure continues iteratively until the stopping criterion is satisfied.

\section{B. Archived Multiobjective Simulated Annealing}

Simulated annealing (SA) is inspired by the physical process of heating and controlled cooling of a material and is a good stochastic local search based method for global optimization. AMOSA is based on the SA and aims to solve the multi-objective optimization problem. It also uses an archive to store the nondominated solutions.

A number of $\gamma \times S L(\gamma>1)$ solutions are generated initially, where SL is the the maximum size to which the archive may be filled. A simple hill-climbing technique is applied to these solutions until a number of $H L$ nondominated solutions are stored in the archive. One of the solutions, called current-pt, is selected randomly from the archive as the initial solution at temperature Temp $=\operatorname{Tmax}$. Then a new solution, called new-pt, is generated by perturbing the current-pt. The domination status of new-pt is checked with the current-pt and solutions in the archive respectively. Three different cases may arise based on the domination relations between current-pt and new-pt. A concept based on amount of dominance is developed to calculate the acceptance probability of a new solution to distinguish between "more dominated" solutions from "less dominated" solutions. During the process, whenever the number of nondominated solutions in the archive exceeds $S L$, clustering technique is enforced to reduce the size of archive to $H L$ in order to keep good diversity of the nondominated solutions.

The above process is repeated iter times for each temperature Temp. Temperature is then reduced to $\alpha \times T e m p$, where $\alpha$ representes the cooling rate with value between 0 and 1. The algorithm stops after the temperature reaches its minimum limit, Tmin.

\section{PROBLEM FORMULATION}

In this paper, the time period for the optimal generation scheduling problem is 24 hours. The proposed two-phase approach attempts to minimize the operation cost and emission while satisfying different system and operating constraints.

\section{A.Objective Functions}

1) Operation cost, including fuel costs, startup costs, and shutdown costs for the entire period is given by:

$$
\begin{aligned}
& F_{0}=\sum_{i=1}^{T} \sum_{i=1}^{N G}\left[F C_{i t}+S T C_{i t}+S D C_{i t}\right] \\
& F C_{i t}=\left(a_{i}+b_{i} P_{i t}+c_{i} P_{i t}^{2}\right) U_{i t} \\
& S T C_{i t}=S T_{i t}\left(1-U_{i(t-1)}\right) U_{i t} \\
& S D C_{i t}=S D_{i t}\left(1-U_{i t}\right) U_{i(t-1)} \\
& S T_{i t}=\left\{\begin{array}{lll}
H S C_{i} & \text { if } & h_{i t}^{\text {off }} \leq C S H_{i}+M D_{i} \\
C S C_{i} & \text { if } & h_{i t}^{\text {off }}>C S H_{i}+M D_{i}
\end{array}\right.
\end{aligned}
$$

2) We only consider the $\mathrm{NO}_{x}$ emission. However, other emissions could be included as well. The form of the $\mathrm{NO}_{\mathrm{x}}$ emission function model depends on the parameter estimating techniques used to approximate the amount of $\mathrm{NO}_{\mathrm{x}}$ emission $[7,8,9]$. In this paper, the total $\mathrm{NO}_{\mathrm{x}}$ emission produced by all units in $T$ hours is expressed by a combination of polynomial and sinusoidal terms of the following form [7]:

$$
E=\sum_{t=1}^{T} \sum_{i=1}^{N G}\left(\alpha_{i}+\beta_{i} P_{i t}+\gamma_{i} P_{i t}^{2}+\varsigma_{i} \sin \left(\tau_{i} P_{i t}\right) U_{i t}\right.
$$




\section{B. Constraints}

1) Power balance:

$$
\sum_{i=1}^{N G} P_{i t}=D_{t}
$$

2) Spinning reserve:

$$
\sum_{i=1}^{N G} P_{i}^{\max } U_{i t} \geq D_{t}+R_{t}
$$

3) Power generation limits:

$$
P_{i}^{\min } U_{i t} \leq P_{i t} \leq P_{i}^{\max } U_{i t}
$$

4) Minimum up/down time:

$$
\begin{aligned}
& \left(h_{i(t-1)}^{o n}-M U_{i}\right)\left(U_{i(t-1)}-U_{i t}\right) \geq 0 \\
& \left(h_{i(t-1)}^{o f f}-M D_{i}\right)\left(U_{i t}-U_{i(t-1)}\right) \geq 0
\end{aligned}
$$

5) Ramp up/down rate:

$$
\begin{array}{r}
P_{i t}-P_{i(t-1)} \leq\left[1-U_{i t}\left(1-U_{i(t-1)}\right)\right] R U_{i}+U_{i t}\left(1-U_{i(t-1)}\right) P_{i}^{\min } \\
P_{i(t-1)}-P_{i t} \leq\left[1-U_{i(t-1)}\left(1-U_{i t}\right)\right] R D_{i}+U_{i(t-1)}\left(1-U_{i t}\right) P_{i}^{\min }
\end{array}
$$

\section{PROPOSED APPROACH}

Details related to the two phases of the algorithm are outlined in this section.

\section{A. First Phase (hourly schedules)}

The hourly optimal generation scheduling problem, which is addressed in the first phase, is formulated as follows:

Minimize $F_{t}, E_{t}$,

where,

$$
\begin{aligned}
& F_{t}=\sum_{i=1}^{N G}\left[F C_{i}\left(P_{i t}\right)+S T C_{i t}+S D C_{i t}\right] \\
& E_{t}=\sum_{i=1}^{N G}\left[10^{-2}\left(\alpha_{i} \cdot P_{i t}^{2}+\beta_{i} \cdot P_{i t}+\gamma_{i}\right)+\varsigma_{i} \sin \left(\tau_{i} P_{i t}\right)\right]
\end{aligned}
$$

Subject to power balance (7), spinning reserve (8) and power generation limits (9).

In this phase, MOEA is applied separately $T$ times, once for each hourly time interval $t=1,2, \ldots T$. Real valued representation is used here to determine the actual power output of each generation unit. As shown in Fig. 1, each solution in this phase is a row vector of length $N G$, whose $i^{\text {th }}$ entry corresponds to the output of unit $i$ during the time interval $t$ under consideration. At any given time $t$, there are npop such solutions in the population.

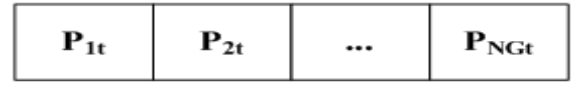

Fig. 1. Solution encoding for hourly dispatch schedule

It is likely that, the initial randomly generated solutions, as well as those created offspring through crossover and mutation, would violate the generation limits constraints and

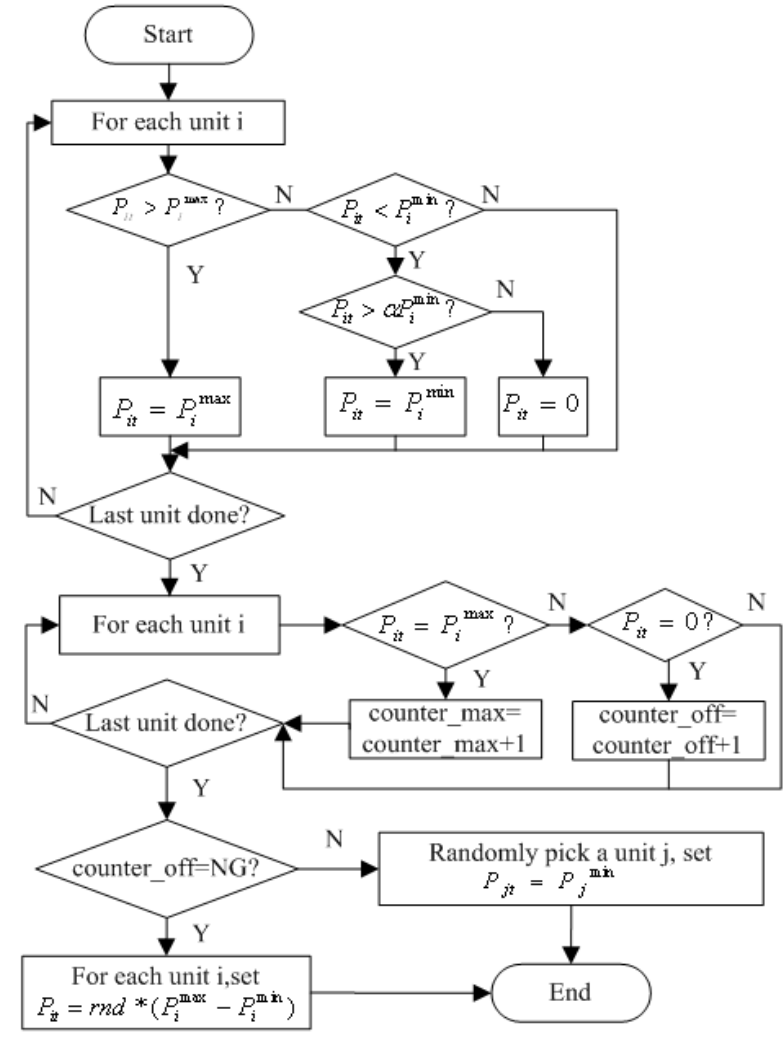

Fig. 2. Generation limits repair scheme

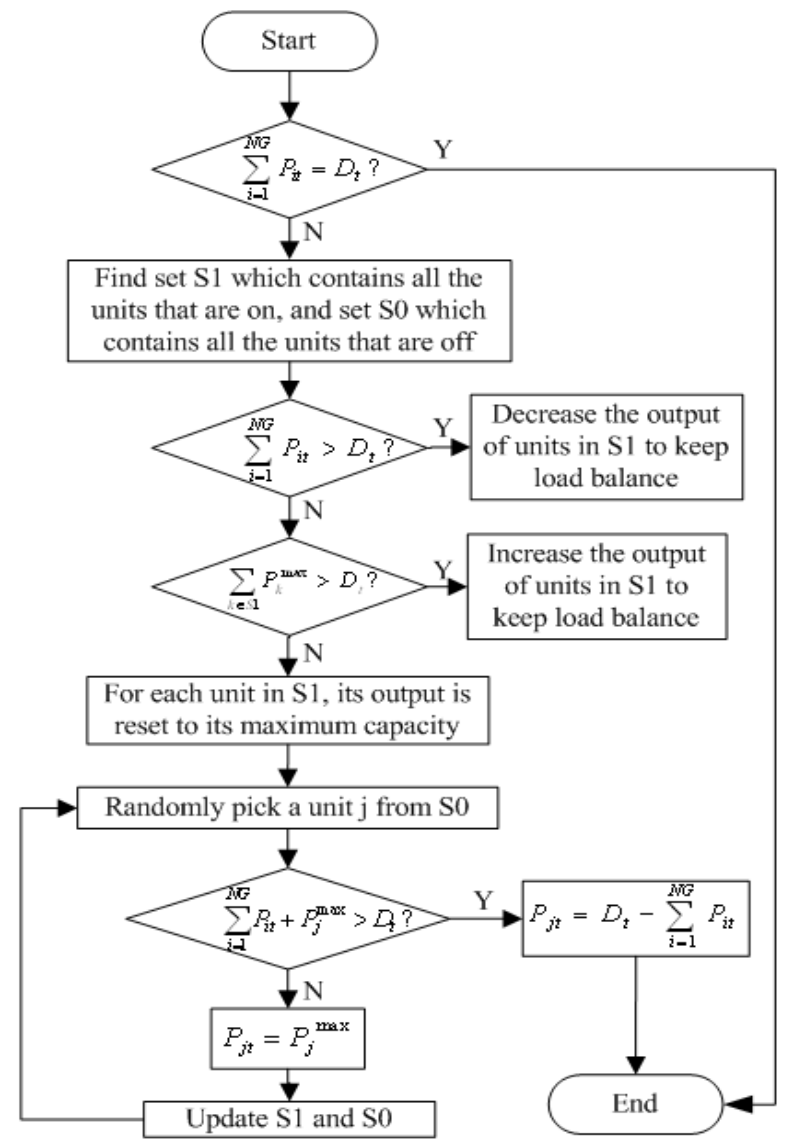

Fig. 3. Load balance repair scheme 
load balance requirement. To make a correction of these infeasible solutions, a repair scheme has been applied to the offspring (or new solutions) that are generated in each iteration. Fig. 2 shows the generation limit repair scheme for three possible situations, where generation limit violation can occur. Fig. 3 shows how the load balance repair scheme works.

\section{B. Second Phase (T-period schedules)}

In the second phase the overall optimal schedule for each hour of the entire $T$ periods can be obtained by assembling the hourly solutions from the first phase. The mathematical formulation of the second phase problem is given below:

Minimize operation cost (3), emission (6), Subject to minimum up/down time (10), (11) and ramp up/down rate (12), (13).

As shown in Fig. 4, the schedule of generation for the entire period of $T$ intervals is represented by a row vector of $T$ indexes, which are integers between 1 and nрор. The $t$ th element in this row vector corresponds to one of the nроp hourly-optimal solutions in hour $t$ obtained during the first phase. For example, if the vector $\left[\begin{array}{llll}15 & 36 & \ldots & 67\end{array}\right]$ were to represent the generation schedule for the entire duration of $T$ periods, it would include the $15^{\text {th }}$ solution obtained during the first phase, for the first hour, the $36^{\text {th }}$ solution for the second hour, and the $67^{\text {th }}$ solution for the last $(t=24)$ hour, as specified in Fig. 1. The initial solution vectors are generated by randomly selecting one index between 1 and npop for each hour.

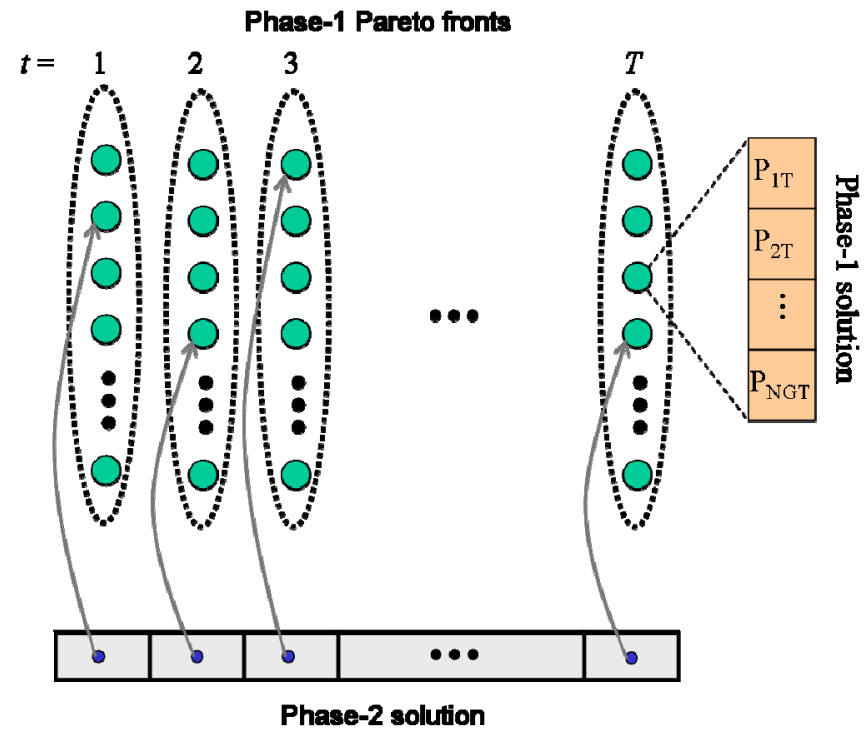

Fig. 4. Solution encoding scheme during the second phase

\section{NUMERICAL SIMULATIONS}

To investigate the performance of the proposed approach, algorithms using SPEA2 and AMOSA were implemented in C language and were applied on a 10-unit system. The system data are based on [10]. The spinning reserve at each hour was kept at $10 \%$ of the load demand and the shutdown cost for each unit was neglected as in [10].

In the first phase, the population size for SPEA2, and the archive size of AMOSA are set to 200. For SPEA2, other parameter settings are: maximum iterations $=200$, probability of crossover $=0.8$, probability of mutation $=0.1$, and for AMOSA, other parameter settings are: the maximum temperature $\operatorname{Tmax}=100$, the minimum temperature $\operatorname{Tm} \min =10^{-7}$, mutation rate $=0.1$, the hard limit of the archive size $H L=200$, the soft limit of the archive size $S L=250$, and the inner maximum iterations $=500$.

Due to the stochastic nature of our approach, a total of 20 independent runs, comprising of both phases, were carried out. Fig. 5 (a-b) show the Pareto fronts for the first hour obtained by SPEA2 and AMOSA respectively based on the results from one sample run. It can be clearly seen that SPEA2 provides a better Pareto-front than AMOSA based on the two criteria of multi-objective optimization: convergence and diversity. In fact, AMOSA has failed to provide the low cost solutions to the problem. Thus, SPEA2 is selected for the second phase.

In the second phase, parameters used for SPEA2 are: population size $=300$, maximum iterations $=500$, probability of crossover $=0.8$, probability of mutation $=0.05$. Two-point

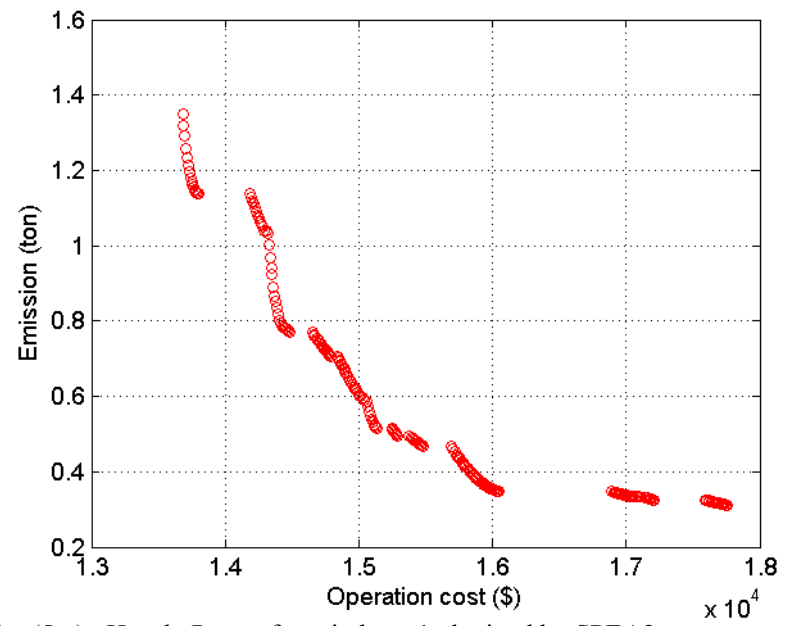

Fig. (5-a). Hourly Pareto front in hour 1 obtained by SPEA2

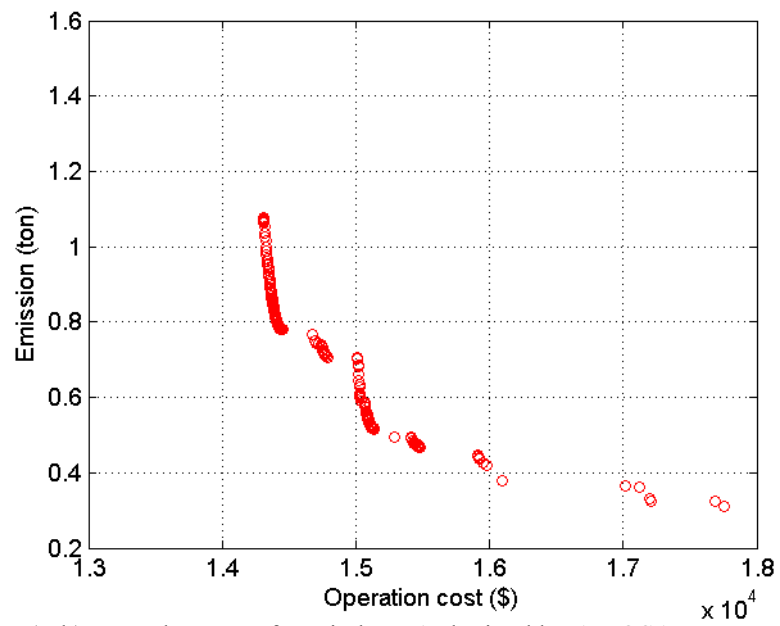

Fig. (5-b). Hourly Pareto front in hour 1 obtained by AMOSA 
crossover and bitwise Gaussian mutation are used. In the twopoint crossover, after the two crossover points are selected, indexes between the two points are swapped between the parents to create two offspring. In the bitwise Gaussian mutation, each element (the solution index corresponding one Pareto-optimal solution in one hour in the first phase) of the row vector representing generation schedules for $T$ periods, is tested with a small probability to decide whether it should be replaced with a new solution index, which is a randomly normally-distributed integer number between 1 and npop.

Fig. 6. shows a sample Pareto front for the 24-hour generation scheduling, which were obtained from the second phase of a sample run of SPEA2. The total operation cost for 24 hours has a wide range from $\$ 570,510$ to $\$ 673,480$, corresponding to the emission's range from 42.17 to 22.88 ton, which gives the decision maker more operating feasibility according to the realistic system conditions.

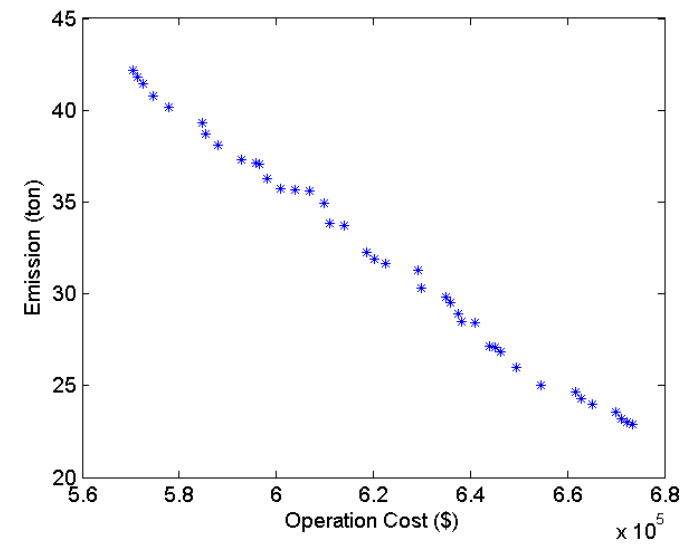

Fig. 6. 24-hour Pareto front obtained by SPEA2

Table I shows the minimum, maximum and the median values of each objective that were found by merging the Pareto optimal solutions of all 20 runs. The small variation between the values of the best and worst boundary solutions proves the robustness of the proposed approach.

TABLE I

Performance of The Proposed Approach For The 10-Unit System (20 RUN AVERAGE)

\begin{tabular}{ccccccc}
\hline \hline & \multicolumn{3}{c}{ Operation Cost (\$) } & \multicolumn{3}{c}{ Emission (ton) } \\
\cline { 2 - 7 } & Min & Max & Median & Min & Max & Median \\
\hline Best & & & & & & \\
Operation & 570,510 & 570,522 & 570,516 & 42.10 & 42.17 & 42.13 \\
Cost & & & & & & \\
Best & 673,467 & 673,480 & 673,474 & 22.88 & 22.95 & 22.91 \\
Emission & & & & & & \\
\hline \hline
\end{tabular}

\section{CONCLUSIONS}

Novel two-phase multi-objective optimization approaches are presented to solve the optimal generation scheduling problem with the environmental considerations. SPEA2 and AMOSA are applied to simultaneously minimize the operation cost and emissions. In the second phase, SPEA2 is used again to get the 24-hour generation schedule with the minimum up/down time and ramp up/down rate constraints enforced. Future research will include modifications to the AMOSA method to improve its performance in the first phase and then applying it to second phase. Work on application of NSGA-II for this problem is under progress and will be reported in a separate paper in the future. Also, these techniques will be applied to larger system to test their performance.

\section{APPENDIX. LIST OF SYMBOLS}

$\alpha_{i}, \beta_{i}, \gamma_{i}, \varsigma_{i}, \tau_{i} \quad$ Characteristic coefficients of unit $i$ 's emission

$a_{i}, b_{i}, c_{i} \quad$ Characteristic coefficients of unit $i$ 's fuel cost

$h_{i t}^{\text {off }} \quad$ Number of continuous time intervals that a unit $i$ has remained OFF before current period $t$

$t \quad$ Index of time period

$C_{i} \quad$ Cold start cost of unit $i$

$\mathrm{CSH}_{i} \quad$ Cold start hours of unit $i$

$D_{t} \quad$ System load demand in period $t$

$E \quad$ Total emission produced by all units for the entire time interval $T$

$E_{t} \quad$ Emission produced by all units in period $t$

$F \quad$ Total operation cost for the entire time interval $T$ considering emission allowance trading

$F C_{i t} \quad$ Fuel cost of unit $i$ in period $t$

$F_{t} \quad$ Operation cost of all units in period $t$

$H S C_{i} \quad$ Hot start cost of unit $i$

$M D_{i} \quad$ Minimum down time of unit $i$

$M U_{i} \quad$ Minimum up time of unit $i$

$N G \quad$ Number of units

$P_{i t} \quad$ Power output in period $t$

$P_{i}^{\max } \quad$ Maximum generation limit of unit $i$

$P_{i}^{\min } \quad$ Minimum generation limit of unit $i$

$R_{t} \quad$ Reserve requirement in period $t$

$R D_{i} \quad$ Ramp-down limit of unit $i$

$R U_{i} \quad$ Ramp-up limit of unit $i$

$S D C_{i}$

Shutdown cost of unit $i$ in period $t$

$S T C_{i} \quad$ Startup cost of unit $i$ in period $t$

So Set of off-line units

S1 Set of on-line units

$T \quad$ Entire time interval, in this paper, 24 hours

$U_{i t} \quad 0 / 1$ variable which states $\mathrm{OFF} / \mathrm{ON}$ status of unit $i$ in period $t$

\section{REFERENCES}

[1] A.A. El-Keib, H. Ma and J.L. Hart, "Economic dispatch in view of the Clean Air Act of 1990," IEEE Trans. Power Syst., vol. 9, no. 2, pp. 972-978, May 1994. 
[2] I. Das and J. Dennis, "A closer look at drawbacks of minimizing weighted sums of objectives for pareto set generation in multicriteria optimization problems," Structural Opt., vol. 14, no. 1, pp. 163-69, 1997.

[3] E. Zitzler, M. Laumanns, and L. Thiele, "SPEA2: Improving the strength pareto evolutionary algorithm for multiobjective optimization," Evolutionary Methods for Design, Optimisation and Control, CIMNE, Barcelona, Spain, 2002

[4] S. Bandyopadhyay, S. Saha, U. Maulik, and K. Deb, "A Simulated Annealing-Based Multiobjective Optimization Algorithm: AMOSA," IEEE Trans. Evol. Comput., vol. 12, no. 3, pp. 269-283, June 2008.

[5] S. Das and B. Panigrahi, "Multi-objective Evolutionary Algorithms," Encyclopedia of Artificial Intelligence, (Eds. J. R. Rabuñal, J. Dorado \& A. Pazos), Idea Group Publishing, vol. 3, pp 1145-1151, 2008.

[6] K. Deb, Multiobjective Optimization Using Evolutionary Algorithms. New York: Wiley, 2001.

[7] I. H. Talaq, F. El-Hawary, and M. E. El-Hawary. "A Summary of Environmental/Economic Dispatch Algorithms," IEEE Trans. Power Syst., vol. 9, no. 3, pp. 1508-1516, 1994.

[8] R. Yokoyama, S. H. Bae, T. Morita, and H. Sasaki, "Multiobjective generation dispatch based on probability security criteria," IEEE Trans.Power Syst., vol. 3, pp. 317-324, Feb. 1988.

[9] R.T.F. Ah King, H.C.S. Rughooputh, K. Deb, "Stochastic evolutionary multiobjective environmental/economic dispatch," in Proceedings of the IEEE Congress on Evolutionary Computation. Vancouver, BC, Canada, pp. 946-953, July 2006.

[10] S. A. Kazarlis, A. G. Bakirtzis, and V. Petridis, "A genetic algorithm solution to the unit commitment problem," IEEE Trans. Power Syst., vol. 11, pp. 83-91, Feb. 1996.

Dapeng Li received the B.S. in Electrical Engineering from Xi'an University of Technology, Xi'an, China in 2001 and the M.S. in Electrical Engineering from Shanghai University, Shanghai, China in 2004. Currently, he is working toward the Ph.D. degree in the Department of Electrical and Computer Engineering at Kansas State University, Manhattan, KS, USA. His research interests are in the areas of computational intelligence applications in power systems and power markets.

Sanjoy Das received his Ph.D. in Electrical Engineering from Louisiana State University, Baton Rouge, in 1994. Between 1994 and 1997 he received his postdoctoral training from the University of California, Berkeley and the Smith-Kettlewell Institute, San Francisco. He has also worked as a research scientist at ITT systems. Since 2001, he has been in the Electrical and Computer Engineering Department at Kansas State University, where he is currently an Associate Professor. His research interests include evolutionary algorithms, multi-objective optimization, and machine learning.

Anil Pahwa received the B.E. (honors) degree in electrical engineering from Birla Institute of Technology and Science, Pilani, India, in 1975, the M.S. degree in electrical engineering from University of Maine, Orono, in 1979, and the Ph.D. degree in electrical engineering from Texas A\&M University, College Station, in 1983.

Since 1983, he has been with Kansas State University, Manhattan, where presently he is a Professor in the Electrical and Computer Engineering Department. He worked at ABB-ETI, Raleigh, NC, during sabbatical from August 1999 to August 2000. His research interests include distribution automation, distribution system planning and analysis, distribution system reliability, and intelligent computational methods for distribution system applications.

Dr. Pahwa is a member of Eta Kappa Nu, Tau Beta Pi, ASEE, and Fellow of IEEE. 